\title{
Ecthyma Gangrenosum: Escherichia coli or Pseudomonas aeruginosa?
}

\author{
Mohamed Abbas ${ }^{1,2 *}$, Stéphane Emonet ${ }^{3}$, Thilo Köhler ${ }^{1,4}$, Gesuele Renzi ${ }^{3}$, \\ Christian van Delden ${ }^{1}$, Jacques Schrenzel ${ }^{3}$ and Bernard Hirschel ${ }^{1}$ \\ 'Division of Infectious Diseases, Department of Medical Specialties, Geneva University Hospitals, Geneva, Switzerland, \\ ${ }^{2}$ Infection Control Programme, Geneva University Hospitals, Geneva, Switzerland, ${ }^{3}$ Bacteriology Laboratory, Division of \\ Infectious Diseases, Geneva University Hospitals, Geneva, Switzerland, ${ }^{4}$ Department of Microbiology and Molecular \\ Medicine, University of Geneva, Geneva, Switzerland
}

OPEN ACCESS

Edited by: Leonard Peruski,

US Centers for Disease Control and Prevention (CDC), United States

Reviewed by:

Miklos Fuzi,

Semmelweis University, Hungary Devendra Hiraman Dusane, Ohio State University, United States

*Correspondence:

Mohamed Abbas

mohamed.abbas@hcuge.ch

Specialty section:

This article was submitted to Infectious Diseases,

a section of the journal

Frontiers in Microbiology

Received: 29 December 2016

Accepted: 12 May 2017

Published: 30 May 2017

Citation:

Abbas M, Emonet S, Köhler T,

Renzi G, van Delden C, Schrenzel J and Hirschel B (2017) Ecthyma Gangrenosum: Escherichia coli or

Pseudomonas aeruginosa?

Front. Microbiol. 8:953.

doi: 10.3389/fmicb.2017.00953
Background: Ecthyma gangrenosum (EG) are necrotic lesions that develop in the context of Pseudomonas aeruginosa bacteremia. Isolated reports describe EG in the setting of non-Pseudomonal infections. In a patient with EG, initial blood cultures showed Escherichia coli, and almost occulted P. aeruginosa bacteremia. Based on the clinical picture we suspected preponderant $P$. aeruginosa bacteremia, outgrown by concomitant low-grade $E$. coli bacteremia in the blood culture vials.

Methods: We performed quantitative polymerase chain reaction (PCR) assays with specific primers for $P$. aeruginosa and $E$. coli on blood collected at the same time for blood cultures. We also performed quantitative cultures of the strains isolated from the patient's blood.

Results: Quantitative PCR showed that there were $1.5 \times 10^{\mathrm{E}} 7$ copies/milliliter $(\mathrm{ml})$ of $P$. aeruginosa DNA, whereas the quantity of $E$. coli DNA was below the detection limit of $2 \times 10^{\mathrm{E}_{4}}$ copies $/ \mathrm{ml}$. We estimated that there was at least 1000 times more $P$. aeruginosa than $E$. coli. Quantitative cultures showed that $E$. coli grew faster than $P$. aeruginosa.

Conclusion: Our patient with EG had preponderant $P$. aeruginosa bacteremia, that was almost occulted by concomitant low-grade E. coli bacteremia. Quantitative PCR was complementary to blood cultures in the final microbiological diagnosis, and proved beneficial in establishing the etiology of EG. This may question the existence of nonPseudomonal EG, and also shows that blood culture results do not always reflect an "exact picture" of what happens in the patient's blood at the time of sampling. This case illustrates the importance of communication between the clinician and the microbiology laboratory to ensure best possible results.

Keywords: ecthyma gangrenosum, Pseudomonas aeruginosa, molecular diagnosis of infectious diseases, blood cultures, septic shock

\section{INTRODUCTION}

A 53-year-old man, whose medical history is significant for rheumatoid arthritis treated with corticosteroids, followed by methotrexate and adalimumab. He was diagnosed 4 months before his current hospitalization with glioblastoma after experiencing a seizure. He was treated by radiotherapy and temozolomide, and was hospitalized 1 month later for asthenia and 


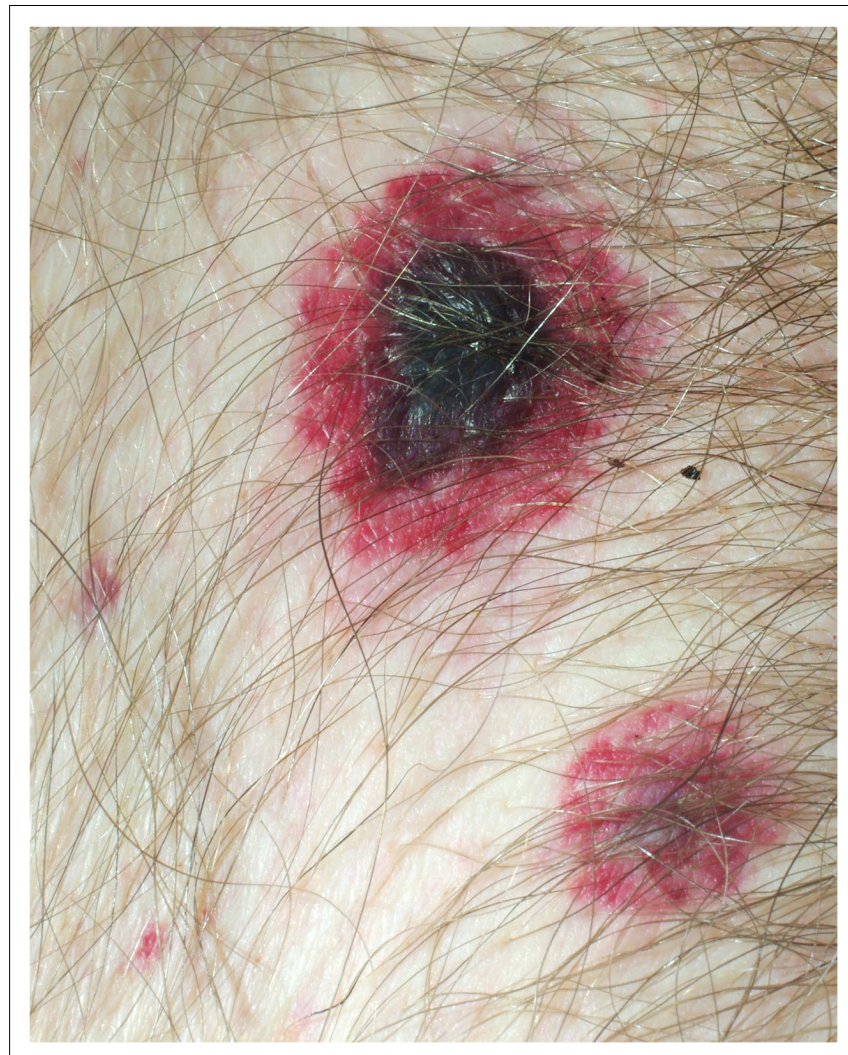

FIGURE 1 | Photograph of a suprapubic ecthyma gangrenosum lesion. Notice the necrotic center of the large lesion, with an erythematous halo. The smaller lesion is presumed to be of an earlier stage.

thrombocytopenia, leading to discontinuation of temozolomide. Brain MRI showed tumor progression, for which pulses of methylprednisolone were given, followed by high doses of dexamethasone.

On day 4 of hospitalization, the patient developed neutropenia, for which Granulocyte Colony Stimulating Factor (G-CSF) was administered. On day 7, he developed neutropenic sepsis for which imipenem/cilastatin was started. On the same day, several skin lesions appeared in the suprapubic area, the right leg, the left popliteal region, and the back (Figure 1). These were purpuric erythematous nodules with a necrotic center, and from 1 to $7 \mathrm{~cm}$ in diameter. The clinical suspicion was EG due to Pseudomonas aeruginosa bacteremia, therefore amikacin was added, but the patient's condition rapidly deteriorated, and he died on day 12 .

Biopsies of the skin lesions were also obtained, showing necrosis of dermis, hypodermis, and basal epidermis, with massive extravasation of red blood cells, and necrotic and thrombosed blood vessels. Vessels situated outside of the necrosis did not show signs of vasculitis. Separation of the epidermis from the dermis was present. Cultures were positive for P. aeruginosa.

The blood cultures revealed Gram-negative rods after $24 \mathrm{~h}$, identified as E. coli the following day. Retrospectivesearch for Pseudomonas in the initial blood cultures allowed identification of one lactose negative colony on the MacConkey agar, which proved to be $P$. aeruginosa by Matrix Assisted Laser Desorption/Ionization Time-of-Flight Mass Spectrometry (MALDI-TOF MS, Microflex ${ }^{\mathrm{TM}}$, Bruker Daltonics, Germany). It was hypothesized that there was a preponderant $P$. aeruginosa bacteremia, causing the EG, but that concomitant low grade $E$. coli bacteremia had outgrown $P$. aeruginosa in the blood culture vials.

To provide evidence for this hypothesis, we performed molecular biology assays and quantitative cultures. Written informed consent to publish this case report anonymously, without identifying information, was provided by the deceased patient's spouse.

\section{Methods}

\section{Genomic DNA Extraction from Whole Blood (EDTA Tube)}

We obtained a whole blood sample drawn on the same day as the first blood cultures, from which we extracted genomic DNA from four independent 100 microliter $(\mu \mathrm{l})$ aliquots using a DNeasy ${ }^{\circledR}$ Blood and Tissue Kit according to the manufacturer's instructions (Qiagen, Hildesheim, Germany).

\section{Quantitative Polymerase Chain Reaction (qPCR) Assay}

We determined the number of $P$. aeruginosa and $E$. coli genomic copies in the patient's blood by qPCR performed on the genomic DNA preparations, using primers specific for $P$. aeruginosa (rpsL-F: 5'-GCAAGCGCATGGTCGACAAGA-3'/rpsL-R: $\quad 5^{\prime}$ CGCTGTGCTCTTGCAGGTTGTGA-3') (Dumas et al., 2006) and E. coli (UAL1939b: 5'-ATGGAATTTCGCCGATTTTGC-3'/ UAL2105b: 5'-ATTGTTTGCCTCCCTGCTGC-3') (Maheux et al., 2009). The reaction contained $3 \mu \mathrm{l}$ of genomic DNA, primers at $600 \mathrm{nM}$ each and 1x of Quantitect Sybr Green Master Mix (Qiagen) in a total volume of $15 \mu \mathrm{L}$. Duplicate samples were run in a RotorGene 3000 (Corbett Research, Sydney, NSW, Australia) as described previously (Dumas et al., 2006). The amount of genomic DNA copies in the blood samples was calculated by comparison with standard curves of $P$. aeruginosa and E. coli genomic DNA preparations. The specificity of the primers was verified on the $P$. aeruginosa and E. coli genomic DNA preparations (as $P$. aeruginosa culture samples were not amplified using the UAL1939b and UAL2105b (E. coli) primer pair, and E. coli culture samples were not amplified using the rpsL F/R ( $P$. aeruginosa) primer pair).

\section{Quantitative Culture Methods}

We thawed E. coli and $P$. aeruginosa isolates from our patient $(\mathrm{A})$, as well as isolates from unrelated patients $(\mathrm{B}+\mathrm{C})$ to make sure that we were not confronted to strain-specific behavior. Identification was confirmed by MALDI-TOF MS. A loopful of bacteria was inoculated in sterile $\mathrm{NaCl} 0.9 \%$ and diluted successively using McFarland turbidity. To allow quantification of the inoculum, each dilution was streaked on sheep blood agar at the time blood culture vials were inoculated. We seeded 8 milliliters $(\mathrm{ml})$ of $E$. coli and of $P$. aeruginosa suspension from each patient $(\mathrm{A}, \mathrm{B}$, and $\mathrm{C})$ in six different aerobic blood culture vials. We added $4 \mathrm{ml}$ of $E$. coli $(100 \mathrm{cfu} / \mathrm{ml})$ and $4 \mathrm{ml}$ 
of $P$. aeruginosa $(1000 \mathrm{cfu} / \mathrm{ml})$ from each patient $(\mathrm{A}, \mathrm{B}$, and C) to a blood culture vial (total of three vials), placed them into a BACTEC blood culture system (Becton-Dickinson), and recorded time to positivity (TTP). At that point, $100 \mu \mathrm{l}$ of each culture dilutions were spread on MacConkey and blood agars. E. coli (pink colonies) and $P$. aeruginosa (colorless colonies) were quantified by CFU counts after $16 \mathrm{~h}$ of incubation at $37^{\circ} \mathrm{C}$.

\section{Results}

The standard curves for $P$. aeruginosa using the rpsL F/R primer pair showed a linear correlation between the amount of bacteria determined on the genomic DNA extracts and the number of cfu added. Identical results were observed for E. coli using the UAL1939b and UAL2105b primer pair. The four genomic DNA preparations from the patient's blood yielded highly reproducible curves when amplified with the $P$. aeruginosa primer pair, with similar threshold values. The number of genomic copies of $P$. aeruginosa was $1.5( \pm 0.045)^{*} 10^{7} / \mathrm{ml}$. We detected no genomic copies of $E$. coli in sample A after amplification of the genomic DNA preparations using the E. coli primer pair, with a detection threshold of $2^{*} 10^{4}$ copies $/ \mathrm{ml}$ blood. Based on this detection level, we estimated that in the patient's blood, the concentration of $P$. aeruginosa DNA was at least 1000 times more elevated than the concentration of E. coli DNA.

The quantitative cultures showed that the TTP for E. coli was $9-10 \mathrm{~h}$, as compared to $15-16 \mathrm{~h}$ for $P$. aeruginosa. After mixed seeding, using MacConkey agar, isolated $P$. aeruginosa colonies were buried in a mass of E. coli colonies, and could easily have been "missed" if the laboratory technician wasn't specifically alerted to the possibility of their presence. Therefore, despite an initial inoculum of $4000 \mathrm{cfu} / \mathrm{ml}$ of $P$. aeruginosa compared to $400 \mathrm{cfu} / \mathrm{ml}$ of $E$. coli, the latter outcompeted Pseudomonas in the mixed blood culture vial as well as on the MacConkey agar.

\section{BACKGROUND}

Ecthyma gangrenosum (EG) was the name given to skin lesions that "evolved from erythematous macules or vesicles into necrotic ulcers" associated with Bacillus pyocyaneus (former name of $P$. aeruginosa) (Greene et al., 1984). Most reports of EG involve patients with disorders of the innate immune system, such as hematological malignancies or drug-induced neutropenia (Dorff et al., 1971; Greene et al., 1984; Reich et al., 2004). While EG is pathognomonic of $P$. aeruginosa bacteremia, reports of EG or EG-like lesions associated with other microorganisms have recently been extensively reviewed and summarized (Vaiman et al., 2015), and the authors suggest that broad definitions of EG should be adopted. It remains controversial whether EG is exclusively associated with $P$. aeruginosa or not. Our objective was to shed new light on this debate, and to illustrate the importance of communication between the clinician and the microbiology laboratory.

\section{DISCUSSION}

Despite initial blood cultures suggesting mono-bacterial E. coli bacteremia, we confirmed our suspicion of preponderant $P$. aeruginosa bacteremia by demonstrating that the quantity of $P$. aeruginosa DNA was at least 1000 times that of $E$. coli. Experimental quantitative cultures showed that the growth of $P$. aeruginosa was slower than that of $E$. coli when cultivated separately, and was completely overwhelmed by the growth of E. coli when cultivated together. It has previously been reported that $P$. aeruginosa has longer doubling times in the presence of other bacteria, perhaps due to competition for nutrients (Tamagnini and González, 1997).

These results suggest that blood cultures might sometimes yield a biased vision of what is in the patient's blood. Reports of the clinical application of molecular diagnosis of sepsis suggest that the combination of PCR and blood cultures is superior to either technique alone, and may thus be complementary (Lamoth et al., 2010; Lehmann et al., 2010). Real-time PCR identified microorganisms that were unidentified by culture, particularly in patients who were receiving antibiotics at the time of sampling (Lamoth et al., 2010; Pasqualini et al., 2012). Molecular diagnosis of sepsis has limitations that make its implementation in routine diagnostic laboratory methods impossible at the moment (detection thresholds, interpretation of DNAemia) (Emonet and Schrenzel, 2011). Newer methods of molecular diagnosis are being evaluated, such as PCR followed by electrospray ionizationmass spectrometry (PCR/ESI-MS), with promising prospects, yet they also require further evaluation (Vincent et al., 2015).

It is controversial whether ecthyma-like lesions due to pathogens other than $P$. aeruginosa should be called EG. Some believe that EG is "specifically associated with systemic $P$. aeruginosa infection" (Dorff et al., 1971; Greene et al., 1984). For example, Hurwitz states that "it may be better to avoid the name EG for these localized, progressive ulcerative infections because they may not be the result of a bacteremia or septicemia, and they may not be due to a Pseudomonas organism" (Hurwitz, 1987). We believe it is legitimate to ask whether the reported cases of non-Pseudomonal EG cases represent mixed bacteremia with undetected $P$. aeruginosa, and therefore adhere to the original definition (Dorff et al., 1971; Greene et al., 1984; Hurwitz, 1987).

\section{CONCLUDING REMARKS}

This case teaches us that even results from a "gold standard" test may be equivocal, and highlights the added value of a clinical diagnosis to a microbiology laboratory's routine work; regular communication between laboratory and clinician is a win-win situation, which ultimately benefits the patient (Baron et al., 2013).

\section{AUTHOR CONTRIBUTIONS}

All the authors provided substantial contributions to the conception and design of the work. MA and TK designed, 
performed and interpreted the results the quantitative PCR experiments under the supervision of CvD. SE and GR designed, performed and interpreted the results of the quantitative culture experiments under the supervision of $\mathrm{BH}$. All authors participated actively in the interpretation of the microbiological results, as well as the drafting of the manuscript. All authors approved the final version of the manuscript, and agree to be accountable for all aspects of the work in ensuring that questions

\section{REFERENCES}

Baron, E. J., Miller, J. M., Weinstein, M. P., Richter, S. S., Gilligan, P. H., Thomson, R. B., et al. (2013). A guide to utilization of the microbiology laboratory for diagnosis of infectious diseases: 2013 recommendations by the Infectious Diseases Society of America (IDSA) and the American Society for Microbiology (ASM)(a). Clin. Infect. Dis. 57, e22-e121. doi: 10.1093/cid/ cit 278

Dorff, G. J., Geimer, N. F., Rosenthal, D. R., and Rytel, M. W. (1971). Pseudomonas septicemia. Arch. Intern. Med. 128, 591-595.

Dumas, J.-L., van Delden, C., Perron, K., and Köhler, T. (2006). Analysis of antibiotic resistance gene expression in Pseudomonas aeruginosa by quantitative real-time-PCR. FEMS Microbiol. Lett. 254, 217-225. doi: 10.1111/j. 1574-6968.2005.00008.x

Emonet, S., and Schrenzel, J. (2011). How could rapid bacterial identification improve the management of septic patients? Exp. Rev. Anti-Infect. Ther. 9, 707-709. doi: 10.1586/eri.11.84

Greene, S. L., Su, W. P., and Muller, S. A. (1984). Ecthyma gangrenosum: report of clinical, histopathologic, and bacteriologic aspects of eight cases. J. Am. Acad. Dermatol. 11, 781-787.

Hurwitz, R. M. (1987). Ecthyma gangrenosum without bacteremia or necrotic cellulitis: a localized form of septic vasculitis. Arch. Intern. Med. 147:1513.

Lamoth, F., Jaton, K., Prod'hom, G., Senn, L., Bille, J., Calandra, T., et al. (2010). Multiplex blood PCR in combination with blood cultures for improvement of microbiological documentation of infection in febrile neutropenia. J. Clin. Microbiol. 48, 3510-3516. doi: 10.1128/JCM.00147-10

Lehmann, L. E., Hunfeld, K.-P., Steinbrucker, M., Brade, V., Book, M., Seifert, H., et al. (2010). Improved detection of blood stream pathogens by real-time PCR in severe sepsis. Inten. Care Med. 36, 49-56. doi: 10.1007/s00134-0091608-z

Maheux, A. F., Picard, F. J., Boissinot, M., Bissonnette, L., Paradis, S., and Bergeron, M. G. (2009). Analytical comparison of nine PCR primer sets designed to related to the accuracy or the integrity of any part of the work are appropriately investigated and resolved.

\section{FUNDING}

This work was supported by a grant from the Swiss National Science Foundation.

detect the presence of Escherichia coli/Shigella in water samples. Water Res. 43, 3019-3028. doi: 10.1016/j.watres.2009.04.017

Pasqualini, L., Mencacci, A., Leli, C., Montagna, P., Cardaccia, A., Cenci, E., et al. (2012). Diagnostic performance of a multiple real-time PCR assay in patients with suspected sepsis hospitalized in an internal medicine ward. J. Clin. Microbiol. 50, 1285-1288. doi: 10.1128/JCM.06793-11

Reich, H. L., Williams Fadeyi, D., Naik, N. S., Honig, P. J., and Yan, A. C. (2004). Nonpseudomonal ecthyma gangrenosum. J. Am. Acad. Dermatol. 50, S114-S117. doi: 10.1016/j.jaad.2003.09.019

Tamagnini, L. M., and González, R. D. (1997). Bacteriological stability and growth kinetics of Pseudomonas aeruginosa in bottled water. J. Appl. Microbiol. 83, 91-94.

Vaiman, M., Lazarovitch, T., Heller, L., and Lotan, G. (2015). Ecthyma gangrenosum and ecthyma-like lesions: review article. Eur. J. Clin. Microbiol. Infect. Dis. 34, 633-639. doi: 10.1007/s10096-014-2277-6

Vincent, J.-L., Brealey, D., Libert, N., Abidi, N. E., O’Dwyer, M., Zacharowski, K., et al. (2015). Rapid diagnosis of infection in the critically ill, a multicenter study of molecular detection in bloodstream infections, pneumonia, and sterile site infections. Crit. Care Med. 43, 2283-2291. doi: 10.1097/CCM. 0000000000001249

Conflict of Interest Statement: The authors declare that the research was conducted in the absence of any commercial or financial relationships that could be construed as a potential conflict of interest.

Copyright (c) 2017 Abbas, Emonet, Köhler, Renzi, van Delden, Schrenzel and Hirschel. This is an open-access article distributed under the terms of the Creative Commons Attribution License (CC BY). The use, distribution or reproduction in other forums is permitted, provided the original author(s) or licensor are credited and that the original publication in this journal is cited, in accordance with accepted academic practice. No use, distribution or reproduction is permitted which does not comply with these terms. 